\title{
封闭组合电气 GIS 机构箱进水导致的事故分析
}

\section{Accident Analysis of Water Intake in Enclosed Combined Electrical GIS Mechanism Box 桂辉阳}

Huiyang Gui

中国南方电网超高压输电公司天生桥局 中国・贵州 兴义 562400

Tianshengqiao Bureau of UHV Transmission Co., Ltd., China Southern Power Grid, Xingyi, Guizhou, 562400, China
【摘 要】机构箱进水可能导致设备误动、拒动, 增加电力事故事件的发生概率, 影响电网 的安全稳定运行。论文主要分析了鲁西换流站机构箱进水导致的事故, 阐述了事故发生的 原因以及三相不一致动作的原理。

【Abstract】Mechanism box water intake may lead to equipment malfunction and refusal to move, increase the probability of electric power accident, and affect the safe and stable operation of the power grid. This paper mainly analyses the accident caused by the water intake of the mechanism box of western Shandong converter station, and expounds the causes of the accident and the principle of three-phase inconsistent action.

【关键词】全封闭组合电器;机构箱进水;三相不一致;鲁西换流站

【Keywords】fully enclosed combined electrical appliances; mechanism box water inflow; three-phase inconsistency; western Shandong converter station

【DOI】10.36012/peti.v1i1.378

\section{1 全封闭组合电气 GIS 的概述}

随着经济的迅猛发展, GIS 组合电器的使用量每年大约 按 $15 \%$ 的速度增加。这类产品既符合无油化的要求, 又符合 小型化、自动化的要求。在整套设备出厂前,采用了预安装。使 设备更加紧凑, 体积更加小型化, 保护更加可靠, 安装更加方 便。智能电网是当今世界电力系统发展变革的最新动向, 而电 网设备及其智能化是构建坚强智能电网的重要基石。随着中 国电网的快速发展, 对全封闭组合电器设备的智能化提出了 迫切的要求, 技术需求与供给存在较大的差异, 已经成为制约 电网发展的重要因素。GIS 全封闭组合电器是换流站的一个 重要组成部分, 也是换流站综合自动化的一个重要监控对象, 它的运行工况实时监测、二次保护、信号传输以及相应的远方 监控可采用综合自动化装置来实现。全封闭组合电器 GIS 较 常规敞开式开关设备更易维护、设备故障少、运行可靠性高, GIS 的使用能有效减少停电事故, 保证送电的可靠性。比 AIS 价格便宜, 但占地面积很大且带电部分外露较多, 限制了在变 电站面积狭小且环境条件恶劣的地方应用, 随着新一轮的电
网改革潮的开启, GIS 将慢慢取代 AIS 势必成为未来发展的 主流。

\section{GIS 的组成及原理}

各种控制和保护电器(断路器、接地开关、隔离开关、互感 器 (VT 及 CT) 、避雷器和连接母线) 全部封装在接地的金属壳 体内, 壳内充以一定压力的 SF6 气体作为相间及对地的绝缘 组合体称为封闭式组合电器, 封闭式组合电器分为分箱式 (一 相一壳)及共箱式(三相共筒)两种,近几年出现了介于 GIS 及 敞开式电器 AIS (Air Insulated Switchgear)之间的半封闭式组 合电器, 简称 H-GIS (Hybrid-GIS)。分箱式 GIS 相间影响小, 运行中不会出现相间短路故障, 而且带电部分与接地外壳间 采用同轴电场结构, 电场的均匀性问题较易解决, 制造也较方 便; 但是, 钢外壳中感应电流引起的损耗大, 外壳数量及密封 面较多, 增加了制造成本及漏气的概率, 其占地面积和体积也 较大。三相共箱式 GIS 的结构紧凑, 外形尺寸和外壳损耗都较 小; 但是, 其内部电场为三维电场, 电场均匀度问题是个难点, 相间影响大。 
故障分析 Fault Analysis

六氟化硫封闭式组合电器是利用多种电路转换器所形成 的一个闭合电路, 通过各种优化设计了一个有机整体, 在进行 各式各样的电能转换, 以及实际气象监测的过程中通过利用 分离各项异性过滤方式来实现结构化的统一行程。GIS 每个 隔室都装设有吸附剂用于吸附水份和 SF6 气体分解物, 并且 断路器端部设置有灭弧室的检修孔, 灭弧室检修十分便利, 检 修时, 所有的元件 (GCB、DS/ES、Bg 等)都可以单独拆解, 不会 影响相邻功能单元 ${ }^{[1]}$ 。

\section{GIS 机构箱进水导致的事故分析}

全封闭组合电器 GIS 机构箱长期运行或由于家族缺陷, 在大雨过后易导致机构箱进水, 由于机构箱的密封性, 进入其 内部的水不易排出, 聚集在内部的水分受热后形成凝露附着 在各接线头的表面或节点处, 导致二次回路导通造成事故。目 前因为进水导致的事故主要有三相不一致回路导通引起三相 不一致动作、失灵保护动作导致断路器自动分合等。

三相不一致继电器在使断路器发生三相位置不一致的时 候, 应能实现已合闸相立即分闸, 且须有一副常开节点引至汇 控箱的端子排上, 鉴于目前三相不一致继电器的试验性按钮 在现场已不再使用, 不再选用带有试验性按钮的继电器。断路 器设置三相不一致保护跳第一组线卷连接片、跳第二组分闸 线圈连接片。该保护用跳闸出口重动继电器采用启动功率不 小于 $5 \mathrm{~W}$ 、动作电压介于 $(55 \% \sim 65 \%) U_{\mathrm{e}}\left(U_{\mathrm{e}}\right.$ 为额定电压)、动作 时间不小于 $10 \mathrm{~ms}$ 的中间继电器。

断路器本体三相不一致保护时间继电器一般采用时间刻 度范围在 $0.5 ~ 5.0 \mathrm{~s}$ 内连续可调, 刻度误差与时间整定值静态 偏差不大于 $\pm 0.1 \mathrm{~s}$ 的高精度时间继电器, 时间定值不发生偏 移, 且保证在强磁环境下运行不易损坏, 不发生误动、拒动。

断路器本体三相不一致采用每相断路器分闸位置辅助常 闭触点并联及合闸位置辅助常开触点并联，之后再串联启动 时间继电器, 经时间继电器延时启动三相不一致保护继电器, 经三相不一致保护继电器接点接通三相跳闸线圈, 以断开仍 在运行的其他相断路器。根据继电保护双重化配置的反措要 求,三相不一致保护也应实现双重化配置。三相不一致保护的 动作时间应按大于断路器单相跳开后等待单相重合闸的时间 来整定。

2016 年 6 月 28 日,工作站发“5142 断路器非全相报警出 现”,跳开 $500 \mathrm{kV}$ 广西侧第四串联络 5142 开关。

三相不一致动作后经到现场检查一、二次设备均未发现 异常。检修人员对 5142 开关进行检查, 检查发现三相不一致 回路 2 SZB2 自保持继电器吸合并自保持, 说明是三相不一致
回路 2 动作造成开关跳闸。由于开关跳闸前 5142 开关三相都 在合位, 所以判断 5142 开关三相不一致回路 2 误动作。在排 查三相不一致回路的过程中, 发现断开 AK48 和 AK50 接点 将 AK49 悬空后, AK49 端子仍然带有 $110 \mathrm{~V}$ 的正电, 从回路可 以看出, AK49 所接-SDA 的 3-4 接点在开关合位时是闭合 的, 这时三相不一致延时继电器 SJ2 会动作, 并延时合上 1518 接点给三相不一致动作继电器励磁, 造成三相不一致动 作,跳开开关。三相不一致回路如图 1 所示。

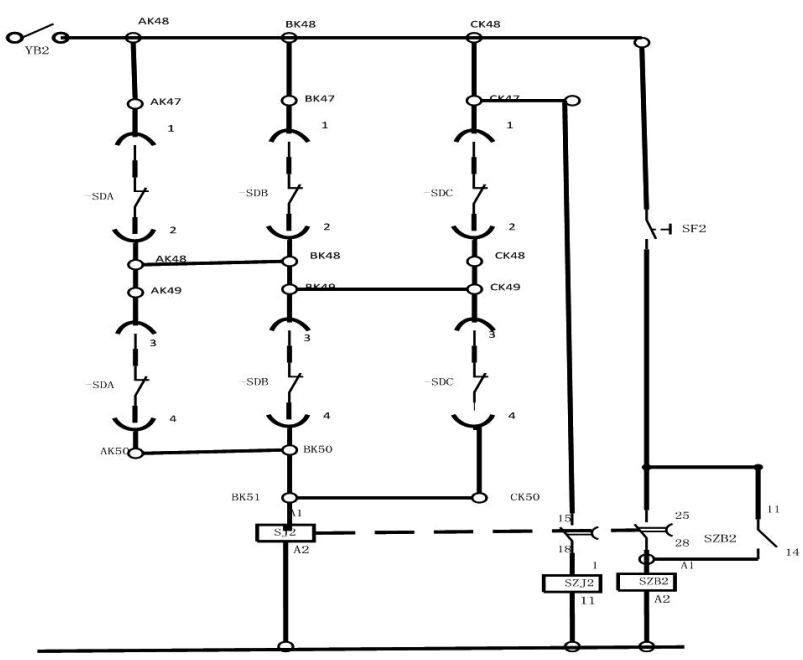

图 1 三相不一致回路

经过对机构箱及二次回路的排查, 发现机构箱中有大量 积水。带电的原因为航空插头受潮造成同插头的信号回路电 压串入控制回路。在对受潮的航空插头进行干燥处理之后, 正 电消失。

\section{4 结语}

全封闭组合电器 GIS 机构箱及汇控箱是整个 GIS 二次回 路的核心,机构箱与汇控柜的外壳的防护等级应不低于 IP54 的要求, 应能防寒、防热、防漏、防水、通风良好, 且应防止雨雪 进人。GIS 长期运行以后易出现外壳锈蚀, 或者防水垫圈腐 化, 为了防止全封闭组合电器长期运行外壳锈蚀或防水垫圈 腐化导致机构箱进水造成事故，运维人员应在设备老化期加 强巡视, 及时发现问题。对于设备存在家族性缺陷而导致的设 备进水, 可对设备进行改造, 如在机构箱或汇控箱外壳加装防 雨罩、在机构箱内装设加热器或除湿机, 以保证柜内湿度不大 于 $70 \%$ 。

\section{参考文献}

[1]注沨,邱毓昌.气体绝缘开关装置(GIS)的近期发展动向 [J].电 网技术,2003(2):54-57. 